\title{
First Principles Calculations of Close-Packed and Doped Carbon Nanotubes*
}

\author{
Noriyuki Ogasawara ${ }^{\dagger}$ and Wataru Norimatsu \\ Department of Applied Chemistry, Graduate School of Engineering, \\ Nagoya University, Furo-cho, Chikusa-ku, Nagoya, Aichi 464-8603, Japan \\ Michiko Kusunoki \\ Environmental Systems and Recycling Science Research Department, EcoTopia Science Institute, \\ Nagoya University, Furo-cho, Chikusa-ku, Nagoya, Aichi 464-8603, Japan \\ (Received 31 January 2012; Accepted 4 June 2012; Published 4 August 2012)
}

\begin{abstract}
The electronic structures of doped carbon nanotubes (CNTs) need to be investigated. We have studied boron and nitrogen-doped, close-packed, aligned, single-walled carbon nanotubes (SWCNTs) using first principles calculations based on density functional theory (DFT). The total energy and density of states (DOS) for doped $(10,0)$ SWCNTs were calculated. From these results, we found that it is more energetically favorable to dope boron atoms than nitrogen atoms. We also found that the electronic structure of close-packed and zigzag-type $(10,0)$ SWCNTs changed to that of a $p$-type semiconductor and metal by doping boron and nitrogen atoms, respectively.

[DOI: $10.1380 /$ ejssnt.2012.411]
\end{abstract}

Keywords: Density functional calculations; Electronic structure; Carbon nanotube; Boron; Nitrogen atom

\section{INTRODUCTION}

Carbon nanotubes (CNTs) have been extensively researched in the many fields since their discovery in 1991 by Ijima [1]. CNTs have several novel properties, such as high strength and good performance as electrical and thermal conductors, and therefore, are expected to be useful in various areas of technology. However, to employ CNTs in electronic devices, it is necessary to control their electronic properties. The structure of CNTs is described by a chiral vector $\mathbf{C}_{\mathbf{h}}$, which connects two crystallographically equivalent sites on the unrolled graphene sheet. $\mathbf{C}_{\mathbf{h}}=n \mathbf{a}_{\mathbf{1}}+m \mathbf{a}_{\mathbf{2}}$, where $n$ and $m$ are integers and $\mathbf{a}_{\mathbf{1}}$ and $\mathbf{a}_{\mathbf{2}}$ are unit vectors of the honeycomb graphene lattice. Thus, each CNT is uniquely labeled by a pair of integers, $(n, m)$ describing its chirality. Using these chiral indices, CNTs have been categorized into three types: armchair, zigzag, and chiral CNTs which are labeled as $(n, n),(n, 0)$, and $(n, m)(n \neq m)$, respectively.

We have reported the selective formation of zigzag CNTs by the surface decomposition of SiC. Moreover, this method enables us to form close-packed CNTs film on $\mathrm{SiC}$ substrates $[2,3]$. Until now, the electronic structure of the isolated, bundled, and doped SWCNTs have

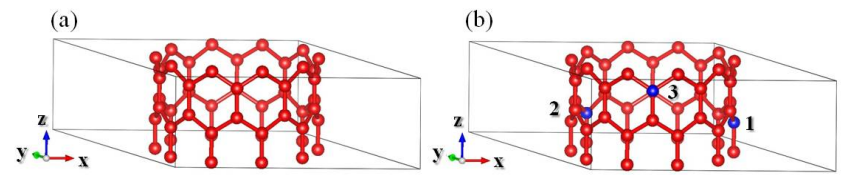

FIG. 1. (a) Red balls represent carbon atoms, and (b) blue balls represent substitutional sites for boron and nitrogen atoms within the supercell indicated by black solid lines. The numbers beside the blue balls are the substitutional order.

\footnotetext{
* This paper was presented at the 6th International Symposium on Surface Science - Towards Nano, Bio and Green Innovation-, Tower Hall Funabori, Tokyo, Japan, December 11-15, 2011.

$\dagger$ Corresponding author: ogasawara.noriyuki@d.mbox.nagoyau.ac.jp
}

been studied by first principles calculations [4-6]. For example, the bandgap decreased with an increase of the Si-concentration [7]. However, the electronic structure of close-packed CNTs film has never been studied. In this study, using first principles calculations, we have investigated the electronic structure of such close-packed SWCNTs and their modification by doping other elements into the SWCNT lattice. In order not to cause too much distortion in the SWCNTs structures, we selected boron and nitrogen as dopants, which have an atomic number close to that of carbon. We calculated the three-dimensional density of states (DOS) of non-doped and doped SWCNTs and found that the electronic structure could be modified by the amount of doping.

\section{COMPUTATIONAL METHOD}

All calculations were performed within the DFT as implemented within the ABINIT code $[8,9]$. We used Troullier-Martins-type (TM) pseudopotentials, PerdewBurke-Ernzerhof (PBE) exchange correlation functionals within a generalized gradient approximation (GGA), and a plane-wave basis with a cutoff energy of 20 hartree [1012]. Periodic boundary conditions were used for the CNTs film with a supercell that has the periodicity of

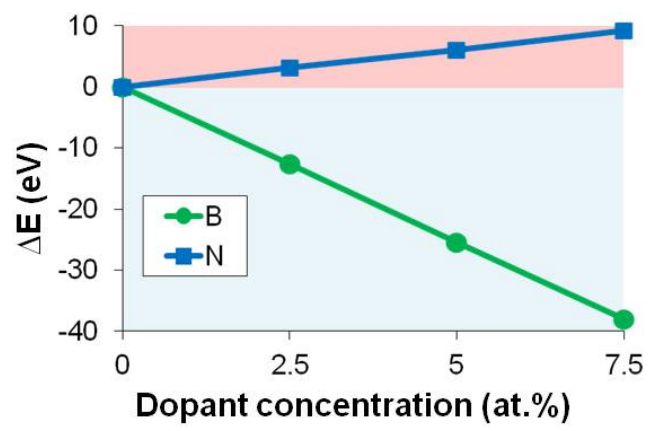

FIG. 2. The results of calculations of $\Delta E$ as a function of dopant concentration. 


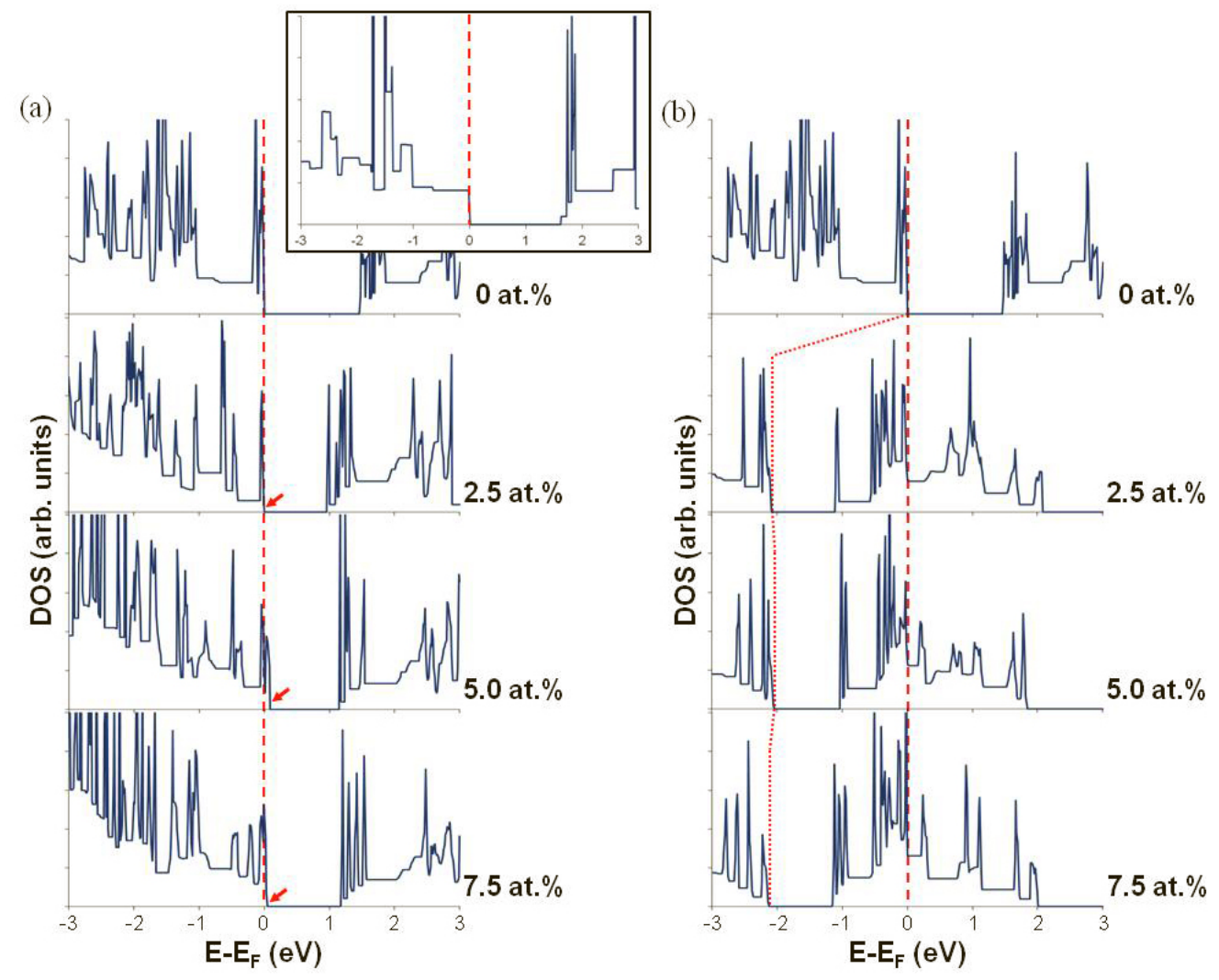

FIG. 3. The comparison of DOS between (a) boron and (b) nitrogen-doped SWCNTs. The Fermi level is shifted to zero and emphasized by red broken lines. The red dotted line in (b) marks the Fermi level of pristine SWCNTs for nitrogen-doped SWCNTs. The result of the DOS for an isolated SWCNT is inserted beside (a).

the SWCNT along the $z$-axis and its size in the $x y$ plane was varied to optimize the intertube distances. The structual optimization was carried out until the forces on all atoms were less than $1.0 \times 10^{-4}$ hartree/Bohr, using the Broyden-Fletcher-Goldfarb-Shanno (BFGS) algorithm [13-16]. For the electronic structure calculations, we used the Monkhorst-Pack scheme to sample the Brillouin zone with a mesh of $3 \times 3 \times 6 k$-points [17].

\section{GEOMETRICAL STRUCTURES}

Before proceeding, we should comment on the structures of SWCNTs and the arrangement of the substituted atoms. In this study, we suppose that the object is an aligned, close-packed, zigzag CNTs film produced by surface decomposition of SiC. Hence, we focused on $(10,0)$ CNTs. Figure 1(a) shows the supercell containing 40 carbon atom sites and Fig. 1(b) shows the arrangement of the substituted boron and nitrogen atoms in the low doping concentration. The intertube distances after the structural optimization are $3.77 \AA$, highly consistent with our experimental results [18]. In order to allow a concise discussion, we define the labels of nanotubes in the following manner. While CNT represents pristine SWCNTs, B ${ }_{1}$ CNT represents boron-doped SWCNTs with one boron atom per supercell, corresponding to a boron concentration is 2.5 at.\%. Similarly, $\mathrm{B}_{2} \mathrm{CNT}, \mathrm{B}_{3} \mathrm{CNT}$, $\mathrm{N}_{1} \mathrm{CNT}, \mathrm{N}_{2} \mathrm{CNT}$, and $\mathrm{N}_{3} \mathrm{CNT}$ represent doped SWCNTs with two and three boron atoms, one, two, and three nitrogen atoms per supercell, respectively.

\section{RESULTS AND DISCUSSIONS}

We first show the result of calculations of the total energy versus dopant concentration. To discuss the stability of boron and nitrogen-doped CNTs, we calculated the formation energy of the doped CNTs by comparing the total energy as follows

$$
\Delta E=E\left(\mathrm{X}_{n} \mathrm{CNT}\right)-\frac{40-n}{40} E(\mathrm{CNT})-n E(\mathrm{X}) .
$$

Here, $E(\mathrm{CNT})$ and $E\left(\mathrm{X}_{n} \mathrm{CNT}\right)$ represent the total energies of a pristine and $\mathrm{X}$-doped ( $\mathrm{X}$ is $\mathrm{B}$ or $\mathrm{N}$ ) CNT, respectively, $E(\mathrm{X})$ represents the energy per atom $\alpha$-boron when $\mathrm{X}$ is $\mathrm{B}$ and in nitrogen gas when $\mathrm{X}$ is $\mathrm{N}$, and $n$ is the number of dopant per supercell [19]. Figure 2 shows the result for $\Delta E$ as a function of the dopant concentration. It is clear that the formation energy of the nitrogen-doped CNTs increases slowly with increases in the nitrogen concentration, but the energy of the boron-doped CNTs decreases much faster on increasing the boron concentration. This indicates that the B-doped CNTs are energetically more favorable, while the N-doped CNTs should be relatively difficult to grow from CNTs in a $\mathrm{N}_{2}$ atmosphere.

Next, we show the results of the DOS for pristine, boron-doped, and nitrogen-doped CNTs. Figure 3 shows 


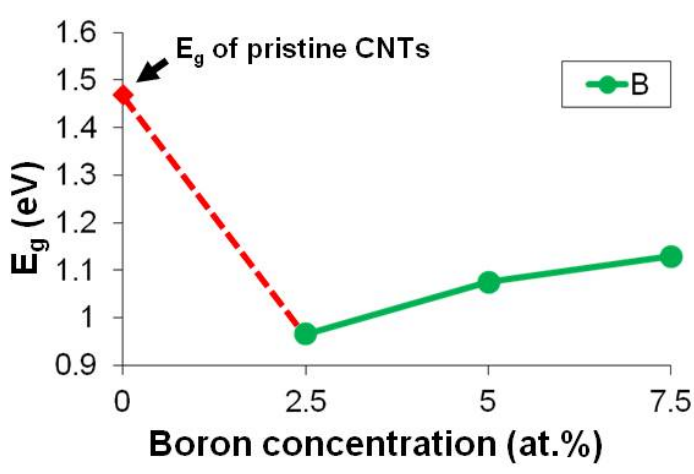

FIG. 4. The results of calculations of band-gap energy $E_{\mathrm{g}}$ as a function of boron concentration.

the DOS as a function of energy relative to the Fermi level for each CNT. As shown in the DOS of 0 at.\%, the Fermi energy is at the top of the valence band, and the band gap is about $1.47 \mathrm{eV}$. The presence of the band gap is consistent with the previous tight binding simulations of isolated $(10,0)$ CNT because the symmetry of $(10,0)$ CNT results in a semiconducting nature [20], though the size of the band gap and the position of the Fermi level are different in the two calculations. We also compare the result with that of the isolated $(10,0) \mathrm{CNT}$, which was inserted in Fig. 3, calculated with the same method. The intertube distance, here, is $11.5 \AA$, which was selected to be large enough to neglect the intertube interactions. The band gap of $1.47 \mathrm{eV}$ for close-packed CNTs is smaller than that of $1.62 \mathrm{eV}$ for isolated ones. In other words, the interactions between the tubes of close-packed CNTs reduce the bandgap. In Fig. 3(a), doping boron atoms introduced additional states and pushed down the Fermi level as indicated by the arrows and then the features of a $p$-type semiconductor appeared. In addition, the energy of the bottom of the conduction band decreased with 2.5 at.\% doping. Boron doping with 2.5 at.\% reduced the bandgap to about $0.95 \mathrm{eV}$, which is about $65 \%$ of the non-doped one. In the reference [6], 2.5 at.\% boron-doped isolated SWNT was $0.44 \mathrm{eV}$, which is about $59 \%$ of the non-doped SWNT. Although the absolute values of the bandgap in the present study and in the reference [6] were different presumably due to the difference in GGA and LDA, the reduction rate of the bandgap is consistent with each other. Thus, 2.5 at.\% boron doping reduces the bandgap by about $40 \%$, regardless of the presence of the intertube interactions. Further doping increased the band-gap energy. Figure 4 shows the doping concentration dependence of the band-gap energy. On the other hand, doping nitrogen atoms strongly pushed up the Fermi level, and the DOS at the Fermi level became large because of the van Hove singularity, indicating metallic characteristics.

From the above results, we come to the conclusion that at the same dopant concentration, nitrogen doping introduces a much higher density of carriers than does boron doping.

\section{CONCLUSIONS}

We have studied the change in the DOS caused by boron and nitrogen doping in the close-packed zigzag CNTs using DFT calculations. From the calculated results of the formation energies of the doped CNTs, we found that doping of boron atoms was favored over nitrogen atoms. From the DOS results, we found that the electronic structure became $p$-type semiconducting and metallic respectively on doping boron and nitrogen atoms in the pristine $(10,0)$ CNTs. Furthermore, for boron doping, the band-gap energy decreased on initial doping, and then reversed to increase with further increases in the dopant concentration.
[1] S. Ijima, Nature 354, 56 (1991).

[2] M. Kusunoki, T. Suzuki, T. Hirayama, and N. Shibata, Appl. Phys. Lett. 77, 531 (2000).

[3] M. Kusunoki , T. Suzuki, C. Honjo, T. Hirayama, and N. Shibata, Chem. Phys. Lett. 366, 458 (2002).

[4] M. U. Kahalya, J. Appl. Phys. 105, 024312 (2009).

[5] X.-L. Sheng, Q.-R. Zheng, Q.-B. Yan, F. Xu, and G. Su, Phys. Lett. A 375, 63 (2010).

[6] T. Koretsune and S. Saito, Phys. Rev. B 77, 165417 (2008).

[7] P. V. Avramov, P. B. Sorokin, A. S. Fedorov, D. G. Fedorov, and Y. Maeda, Phys. Rev. B 74, 245417 (2006).

[8] X. Gonze, et al., Comput. Mater. Sci. 25, 478 (2002).

[9] http://www.abinit.org.

[10] N. Troullier and J. L. Martins, Phys. Rev. B 43, 1993 (1991).
[11] J. P. Perdew, K. Burke, and M. Ernzerhof, Phys. Rev. Lett. 77, 3865 (1996).

[12] J. P. Perdew and Y. Wang, Phys. Rev. B 33, 8800 (1986).

[13] C. G. Broyden, J. Inst. Math. Appl. 6, 76 (1970).

[14] R. Fletcher, Comput. J. 13, 317 (1970).

[15] D. Goldfarb, Math. Comput. 24, 23 (1970).

[16] D. F. Shanno, Math. Comput. 24, 647 (1970).

[17] H. J. Monkhorst and J. D. Pack, Phys. Rev. B 13, 5188 (1976).

[18] M. Kusunoki, C. Honjo, T. Suzuki, and T. Hirayama, Appl. Phys. Lett. 87, 103105 (2005).

[19] A. Masago, K. Shirai, and H. Katayama-Yoshida, Phys. Rev. B 73, 104102 (2006).

[20] R. Saito, G. Dresselhaus, and M. S. Dresselhaus, Physical Properties of Carbon Nanotubes (Imperial College Press, London, 1998). 